\title{
Detection of Planetary Emission from TrES-2 using Spitzer/IRAC
}

\author{
Francis T. O'Donovan ${ }^{1,2}$, David Charbonneau ${ }^{3}$, Joseph Harrington ${ }^{4}$, \\ Sara Seager ${ }^{5}$, Drake Deming ${ }^{1}$, and Heather A. Knutson ${ }^{3}$ \\ ${ }^{1}$ NASA Goddard Space Flight Center, \\ 8800 Greenbelt Rd., Greenbelt, MD 20771, USA \\ email: francis.odonovan@nasa.gov, leo.d.deming@nasa.gov \\ ${ }^{2}$ California Institute of Technology, \\ 1200 E. California Blvd., Pasadena, CA 91125, USA \\ ${ }^{3}$ Harvard-Smithsonian Center for Astrophysics, \\ 60 Garden St., Cambridge, MA 02138, USA \\ email: dcharbonneau@cfa.harvard.edu, hknutson@cfa.harvard.edu \\ ${ }^{4}$ Department of Physics, University of Central Florida, \\ 4000 Central Florida Blvd., Orlando, FL 32816, USA \\ email: jh@physics.ucf.edu \\ ${ }^{5}$ Department of Earth, Atmospheric, and Planetary Sciences, \\ Massachusetts Institute of Technology, \\ 77 Massachusetts Ave., Cambridge, MA 02139, USA \\ email: seager@mit.edu
}

\begin{abstract}
We present here results from observations of TrES-2 made using the Infrared Array Camera on the Spitzer Space Telescope. We monitored this transiting system during two secondary eclipses, when the planetary emission is blocked by the star. The resulting decrease in flux is $0.135 \pm 0.036 \%, 0.245 \pm 0.027 \%, 0.162 \pm 0.064 \%$, and $0.295 \pm 0.066 \%$, at 3.6- $\mu \mathrm{m}$, $4.5-\mu \mathrm{m}, 5.8-\mu \mathrm{m}$, and $8.0-\mu \mathrm{m}$, respectively. We find evidence for a temperature inversion in the atmosphere of TrES-2, which is predicted by Fortney and collaborators based on the proposed importance of $\mathrm{TiO}$ and $\mathrm{VO}$ opacities for this highly irradiated gas giant. We also find the time of the center of the eclipse to be consistent with predictions from transit timing observations of TrES-2. This implies that TrES-2 most likely has a circular orbit, and thus does not obtain additional thermal energy from tidal dissipation of a non-zero orbital eccentricity, a proposed explanation of the large planetary radius.
\end{abstract}

\section{Probing Exoplanet Atmospheres with Spitzer}

The detection of emission from planetary atmospheres is made possible by taking advantage of the enhanced contrast between stars and their planets in the infrared. Spitzer observations of the now numerous nearby transiting hot Jupiters are helping us to understand the thermal dynamics associated with the high insolation on the atmospheres of these planets, and thus why several, such as TrES-2 (see Table 1) have bloated radii.

Several explanations have been proposed for the bloated planets, most of which deal with some additional source of energy combating planetary contraction. Bodenheimer et al. (2001, 2003) proposed that this additional energy could come from tidal damping of a non-zero orbital eccentricity. There has also been a flurry of activity to reconcile atmospheric models with the limited number of Spitzer measurements. Recently, Fortney et al. (2008) supplied a possible piece of the puzzle by proposing that the very highly irradiated hot Jupiters such as TrES-2 will exhibit molecular emission rather than the 
Table 1. TrES-2 System and Planetary Parameters.

Parameter
$V(\mathrm{mag})$
$J(\mathrm{mag})$
$J-K_{s}(\mathrm{mag})$
$R_{\star}\left(R_{\odot}\right)$
$M_{\star}\left(M_{\odot}\right)$
$R_{p}\left(R_{\mathrm{Jup}}\right)$
$M_{p}\left(M_{\mathrm{Jup}}\right)$
$R_{p} / R_{\star}$
$b\left({ }^{\circ}\right)$
$T_{c}(\mathrm{HJD})$
$P(\mathrm{~d})$

$\begin{array}{cr}\text { Value } & \text { Refer } \\ & \\ 11.411 \pm 0.005 & 1 \\ 10.232 \pm 0.020 & 2 \\ 0.386 \pm 0.030 & 2 \\ 1.003 \pm 0.027 & 3 \\ 0.98 \pm 0.11 & 3 \\ & \\ 1.222 \pm 0.038 & 3 \\ 1.28 \pm 0.09 & 3 \\ 0.1253 \pm 0.0010 & 3 \\ 0.8540 \pm 0.0062 & 3 \\ 83.57 \pm 0.14 & 3 \\ & 3 \\ 2,453,957.63479 \pm 0.00038 & 3 \\ 2.470621 \pm 0.000017 & \end{array}$

Notes:

1 O'Donovan et al. (2006)

2 MASS Catalog

3 Holman et al. (2007)

expected absorption at the IRAC wavelengths, a result of a temperature inversion in their atmospheres (see Figure 1 of Fortney et al. 2008).

\section{Observations and Analysis}

We observed TrES-2 during two separate secondary eclipses, and employed all four Infrared Array Camera (IRAC) bandpasses. We obtained 1073 images (10.4s exposures) at $4.5-\mu \mathrm{m}$ and $8.0-\mu \mathrm{m}$ on UT 2006 Nov 30, and 2130/1065 images (1.2s/10.4s) at 3.6- $\mu \mathrm{m}$ and $5.8-\mu \mathrm{m}$ on UT 2007 Aug 16.

We then examined the Basic Calibrated Data images for our observations. These were dark current-subtracted, flat-fielded and corrected for nonlinearity by the Spitzer Science Center. We converted the Julian date corresponding to the mid-exposure of each observation to a Heliocentric Julian date, accounting for the light travel time between the Sun and Spitzer. Using a circular aperture with a 3-pixel radius, we then measured the flux from our target. We selected this radius as the radius that produced the smallest out-of-eclipse photometric rms.

Using code based on Mandel \& Agol (2002), we generated models of the four secondary eclipses (without limb darkening effects). For the 3.6- $\mu \mathrm{m}$ and $4.8-\mu \mathrm{m}$ data, we accounted for the known intra-pixel variability (see, e.g., Knutson et al. 2008) using a quadratic function of the $x$ - and $y$ - pixel positions. We also corrected for a linear trend in the $5.8-\mu \mathrm{m}$ and 8.0- $\mu \mathrm{m}$ fluxes. We computed the best-fit parameters and corresponding errors for our models using the Markov-Chain Monte Carlo method (see, e.g., Winn et al. 2007) with $10^{6}$ steps. The resulting corrected fluxes and best-fit model eclipses are shown in Figure 1 . The time $\left(T_{s}\right)$ of secondary eclipse for these models is that predicted assuming the ephemeris from Holman et al. (2007); we did not allow timing variations. However, we also performed a separate fit of the $8.0-\mu \mathrm{m}$ data, where we allowed such variations.

\section{Preliminary Results}

As is evident from Figure 1, the 3.6- $\mu \mathrm{m}$ and $4.5-\mu \mathrm{m}$ corrected fluxes display linear trends, which must be accounted for in future analysis. Similar trends have been noted in 
the analysis of the Spitzer observations of TrES-4 (Knutson et al., in prep.). Nevertheless, our current results allow us to compare the measured fluxes from TrES-2 with those expected from models. Figure 2 shows a simple model of the TrES-2 planet-star flux ratio, assuming a blackbody temperature of $T_{\text {eq }}=1500 \mathrm{~K}$ for the planet and a Kurucz spectrum at $T_{\mathrm{eq}}=5950 \mathrm{~K}$ for the star. Our IRAC fluxes are overplotted, and appear to be mostly consistent with the model. However, there does appear to be evidence of emission at $4.5-\mu \mathrm{m}$. Such emission would imply a temperature inversion in the atmosphere of TrES-2, as predicted by Fortney et al. (2008).

From our study of the timing variability of the secondary eclipse, we computed a timing offset of $-3.6 \pm 5.8$ minutes, corresponding to an upper limit for the eccentricity of $e \cos \omega=0.00145 \pm 0.00096$, where $\omega$ is the longitude of periastron. Our observations are thus consistent with a negligible orbital eccentricity for TrES-2, which therefore must have another source of energy to explain its bloated radius.

\section{Future Work}

Our future analysis will involve improving our data analysis to reduce the systematic errors, and investigating the linear trend at $3.6-\mu \mathrm{m} \& 4.5-\mu \mathrm{m}$. We will also perform a

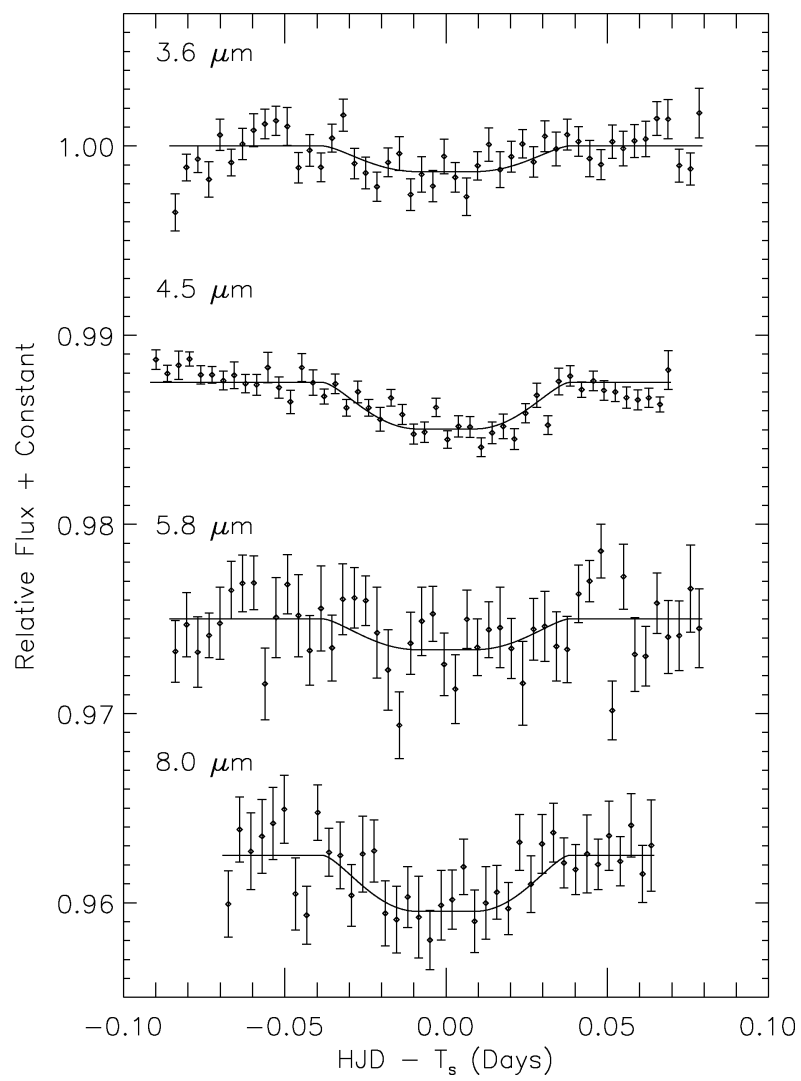

Figure 1. Relative fluxes from the TrES-2 system at the IRAC bandpasses, binned and plotted versus the time from the predicted center of eclipse $\left(T_{s}\right)$. We have accounted for some linear trends and intra-pixel variability in the fluxes. Superimposed are our best-fit model eclipses. 


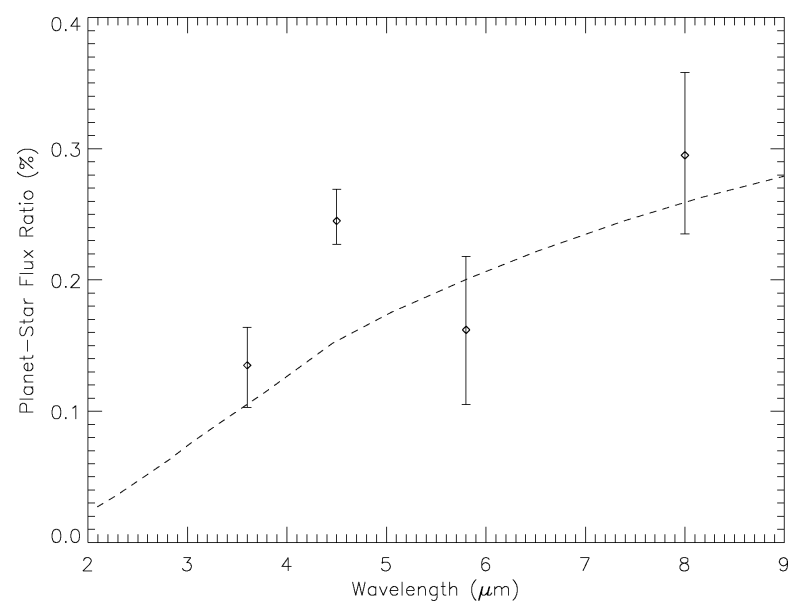

Figure 2. Simple model of TrES-2 planet-star flux ratio, based on a planetary blackbody and Kurucz stellar spectrum, as a function of wavelength. Overplotted are the results of our observations. Three of the data points are consistent with the model, with a $3-\sigma$ emission feature at $4.5-\mu \mathrm{m}$, suggestive of a temperature inversion in the planetary atmosphere.

simultaneous fit of transit timing and eclipse depth for all four bandpasses. Once we have our final results, we will compare these to updated models of exoplanet atmospheres to confirm whether we see evidence for thermal inversion in the atmosphere of TrES-2.

\section{References}

Bodenheimer, P., Laughlin, G., \& Lin, D. N. C. 2003, ApJ, 592, 555

Bodenheimer, P., Lin, D. N. C., \& Mardling, R. A. 2001, ApJ, 548, 466

Fortney, J. J., Lodders, K., Marley, M. S., \& Freedman, R. S. 2008, ApJ, 678, 1419

Holman, M. J., et al. 2007, ApJ, 664, 1185

Knutson, H. A., Charbonneau, D., Allen, L. E., Burrows, A., \& Megeath, S. T. 2008, ApJ, 673, 526

Mandel, K. \& Agol, E. 2002, ApJ, 580, L171

O’Donovan, F. T., et al. 2006, ApJ, 651, L61

Winn, J. N., Holman, M. J., \& Fuentes, C. I. 2007, AJ, 133, 11 